\title{
Le commerce international a-t-il un impact sur la réduction de la pauvreté et des inégalités?
}

\author{
Thierno Diallo, Ph. D., professeur \\ Université du Québec à Chicoutimi \\ Patrice Beaulieu, logisticien \\ Médecins Sans Frontières
}

\section{INTRODUCTION}

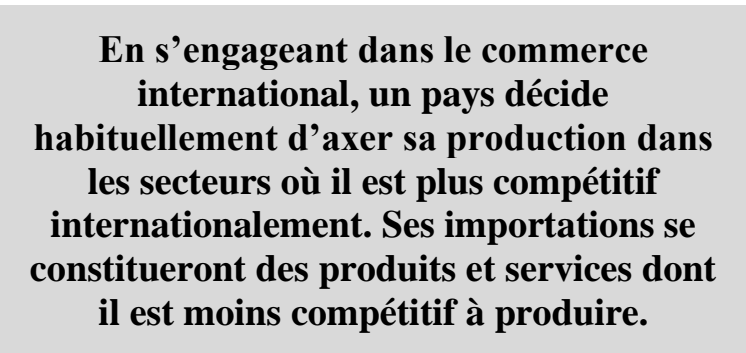

Le commerce international est l'ensemble des activités commerciales requises pour produire, expédier, vendre, et consommer des biens et des services au niveau international. Les théories classiques qui supportent le commerce international remontent à environ 200 ans. Adam Smith formula la théorie des avantages absolus et David Ricardo, quant à lui, celle des avantages comparatifs.

Les pays s'engagent habituellement généralement dans le commerce international pour vendre leurs produits et services sur de nouveaux marchés, ce qui leur permettra de produire plus et ainsi profiter d'économies d'échelles. En s'engageant dans le commerce international, un pays décide habituellement d'axer sa production dans les secteurs où il est plus compétitif internationalement. Ses importations se constitueront des produits et services dont il est moins compétitif à produire.

L'intensification des échanges au niveau international aurait pour effet d'augmenter le niveau de l'emploi dans certains pays et réduire de facto le niveau de pauvreté. Cependant, la notion de pauvreté est difficile à définir, car elle varie selon le contexte que l'on analyse. Par exemple, pour les pays développés: «les individus, familles ou groupes de la population peuvent être considérés en état de pauvreté quand ils manquent des ressources nécessaires pour obtenir l'alimentation type, la participation aux activités et avoir les conditions de vie et les commodités qui sont habituellement ou sont au moins largement encouragées ou approuvées dans les sociétés auxquelles ils appartiennent. » ${ }^{1}$.

Selon le programme des Nations-Unies pour le Développement (PNUD), pour les pays en développement («PED»), voire même des pays les moins avancés («PMA»), la pauvreté se définit comme le manque de ressources matérielles et de conditions de vie nécessaires à la vie ou du moins à une vie digne d'un humain. Il convient de rajouter que la pauvreté est multidimensionnelle, car elle concerne non seulement le revenu des individus, mais également leur santé, nutrition, éducation, et même leurs droits civils et libertés ${ }^{2}$. C'est dans cette optique que le présent article propose une analyse de l'impact du commerce international sur la réduction de la pauvreté et des inégalités.

\section{La pauvreté se définit comme le manque de ressources matérielles et de conditions de vie nécessaires à la vie ou du moins à une vie digne d'un humain. [...] La pauvreté est multidimensionnelle, car elle concerne non seulement le revenu des individus, mais également leur santé, nutrition, éducation, et même leurs droits civils et libertés.}




\section{MODÉLISATION DE LA RELATION ENTRE LE COMMERCE INTERNATIONAL ET LA RÉDUCTION DE LA PAUVRETÉ ET DES INÉGALITÉS}

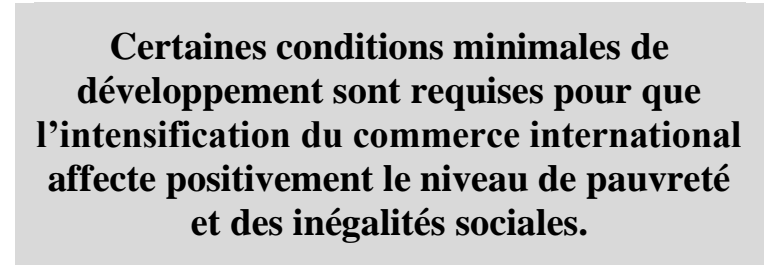

Selon la théorie abordée, il apparaît que le commerce international peut avoir un impact positif sur la réduction de la pauvreté. D'abord, un essor du commerce international, initié par une hausse des exportations, entraînera une croissance et un développement économique. Ensuite, dans le cas où cette croissance est soutenue et inclusive, la pauvreté diminuera. C'est particulièrement le cas des pays moins avancés où la pauvreté est généralisée d'emblée.

Il faut toutefois ne pas sauter aux conclusions. Une hausse des exportations n'est pas nécessairement suffisante pour provoquer une croissance économique soutenue. Il est nécessaire que ces exportations entraînent une hausse des investissements et des progrès technologiques. Dans le cas contraire, les chances que cette croissance économique soit soutenue diminueraient. Considérant que les pays les moins avancés ne disposent généralement pas des capitaux d'investissements nécessaires, une aide devient nécessaire. Le second défi est de s'assurer que cette croissance économique sera inclusive pour réduire les inégalités. Les activités reliées aux exportations devront reposer diverses ressources, et non seulement sur une petite portion de l'économie du pays. Si la croissance liée aux exportations se retrouve ainsi enclavée, elle ne créera pas assez d'activités productrices génératrices de revenus. L'impact sur les revenus et la consommation des populations sera aussi largement atténué, tel qu'illustré à la figure 1 qui présente le modèle théorique de cette étude.

Certaines conditions doivent être respectées pour que l'essor des exportations entraîne une croissance économique soutenue et inclusive ${ }^{3}$. Premièrement, l'accroissement des exportations doit être suivi d'une importation de biens et de services nécessaires au développement et à l'utilisation des capacités productives. Deuxièmement, la réduction de la contrainte de change grâce à l'accroissement des recettes d'exportation ne doit pas être contrebalancée par la réduction des flux d'aide ou l'augmentation des paiements au titre du service de la dette. Troisièmement, l'accroissement des exportations doit renforcer l'accumulation de capital et le progrès technologique dans le pays. Quatrièmement, des interactions positives qui contribuent au développement doivent se mettre en place entre les activités d'exportation en pleine croissance et le reste de l'économie afin d'éviter d'enclaver les activités d'exportations. Donc, l'augmentation du commerce international peut avoir un impact sur la réduction de la pauvreté et des inégalités. Cet impact n'est cependant pas systématique ${ }^{4}$. Certaines conditions minimales de développement sont requises pour que l'intensification du commerce international affecte positivement le niveau de pauvreté et les inégalités sociales.

\section{Figure 1 : Relations entre commerce international, pauvreté et inégalités}

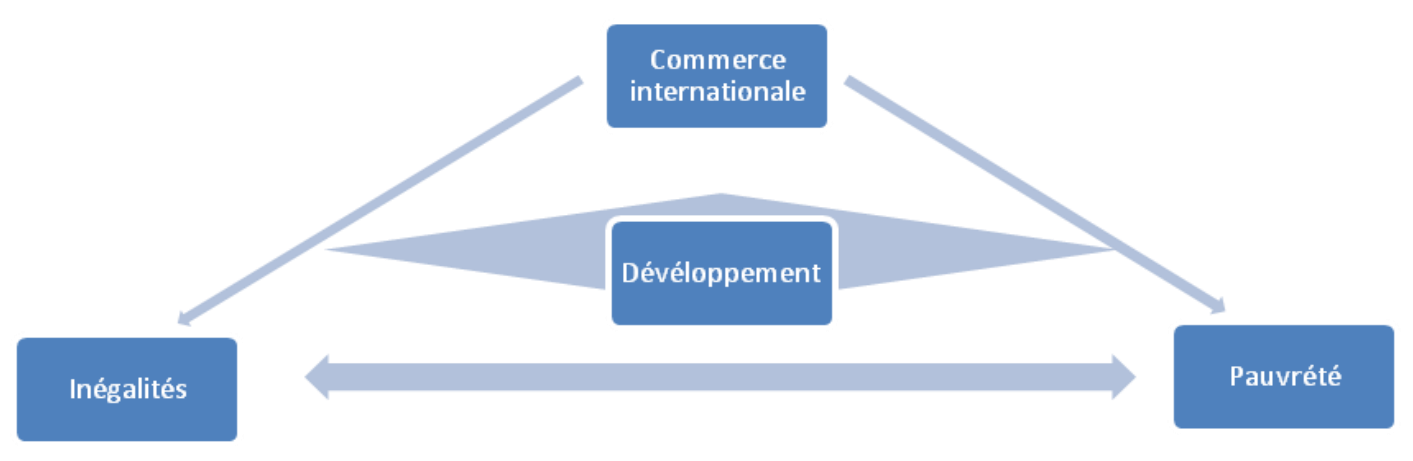




\section{MÉTHODOLOGIE}

\subsection{Choix de l'échantillon et indicateurs}

Pour mener à bien l'analyse empirique de l'impact du commerce international sur la réduction de la pauvreté et des inégalités, nous avons sélectionné cinq pays. Pour chaque pays, nous avons analysé le commerce international, le niveau de développement, le niveau de pauvreté, et le niveau d'inégalité sur une période allant de 1990 à 2005.

Nous avons compilé plusieurs données statistiques provenant de plusieurs sources différentes (ONU, PNUD, OCDE, Banque mondiale, FMI ${ }^{5}$. La disponibilité des données nous a permis de sélectionner les cinq pays suivants :
l'Inde, le Brésil, le Burkina Faso, le Maroc et la Corée du Sud. Nous avons tenté d'inclure différents pays de régions variées et de niveau de développement différents afin de voir si les tendances se répètent d'un ensemble économique régional à l'autre.

Notre objectif étant d'identifier l'existence des liens pour chaque pays sélectionné entre l'évolution de ses échanges internationaux, son niveau de développement économique, ainsi que la réduction de la pauvreté, nous avons dû quantifier ces concepts à l'aide d'indicateurs présentés au tableau 1.

Tableau 1 : Indicateurs empiriques des variables du modèle théorique

\begin{tabular}{|l|l|l|l|}
\hline \multicolumn{1}{|c|}{$\begin{array}{c}\text { Commerce } \\
\text { International }\end{array}$} & Développement & \multicolumn{1}{c|}{ Pauvreté } & \multicolumn{1}{c|}{ Inégalités } \\
\hline $\begin{array}{l}\text { - Exportations } \\
\text { - Balance Commerciale }\end{array}$ & $\begin{array}{l}\text { - Indice du } \\
\text { développement } \\
\text { humain (IDH) }\end{array}$ & $\begin{array}{l}\text { - Pauvreté (ONU) } \\
\text { - Pauvreté extrême (ONU) } \\
\text { - Pauvreté nationale } \\
\text { - Indice HPI-1 }\end{array}$ & $\begin{array}{l}\text { - Indice GINI } \\
\text { - Ratio } 10 \% / 10 \% \\
\text { - Ratio 20\%/20\% }\end{array}$ \\
\hline
\end{tabular}

Pour le commerce international, nous utilisons le volume total annuel d'exportations et la balance commerciale qui est la différence entre la valeur des biens et services qu'un pays exporte et la valeur des biens et services qu'il importe

Pour le développement, l'indicateur retenu est l'Indice de développement humain (IDH). C'est un indice statistique composite, créé par le Programme des Nations unies pour le développement en 1990, évaluant le niveau de développement humain des pays du monde. L'IDH est un indice composite, sans unité, compris entre 0 (exécrable) et 1 (excellent). Il est calculé par la moyenne de trois variables quantifiant respectivement : i) la santé / longévité (espérance de vie à la naissance); ii) le savoir / niveau éducation (taux alphabétisation des adultes et taux brut de scolarisation); et iii) le niveau de vie (logarithme du PIB par habitant en parité du pouvoir d'achat).
La pauvreté, dont la quantification est difficile, voire controversée, est mesurée par quatre indicateurs: i) le seuil de pauvreté défini par l'ONU qui représente le nombre de personnes vivant avec un revenu inférieur à 2 \$ US par jour; ii) le seuil de pauvreté extrême défini par l'ONU qui représente le nombre de personnes vivant avec un revenu inférieur à 1 \$ US par jour; iii) le seuil de pauvreté nationale qui représente le nombre de personnes vivant avec un revenu inférieur au seuil fixé par le pays en question; et iv) l'indicateur de pauvreté humaine (IPH-1) qui mesure l'impact de la pauvreté sur la population.

Le seuil de pauvreté nationale est habituellement calculé selon les coûts des besoins de base dans le pays en question. Le besoin de base le plus important est celui de manger. On estimera ainsi le coût de l'apport calorifique de base pour calculer ce besoin. Par exemple, pour le Burkina 
Faso, il faudrait 2283 calories. Quant à 1'IPH-1, il est basé sur la longévité (\% de décès avant 40 ans), 1 'instruction (\% analphabétisme (incapacité complète à lire et à écrire), et le manque de conditions de vie décentes (\% personnes privées d'accès à l'eau potable, $\%$ personnes privées d'accès aux services de santé, \% enfants de moins de 5 ans souffrant d'insuffisance pondérale).

De plus, dans le but d'améliorer la compréhension du lien entre développement et pauvreté, où certaines disparités apparaissent parfois, nous avons également quantifié les inégalités à l'intérieur des pays sélectionnés. Nous avons donc pris en compte trois indicateurs. D'abord le coefficient de GINI mesure le degré d'inégalité de la distribution des revenus dans une société

\section{ANALYSE DES RÉSULTATS}

Le tableau 2 fournit un résumé des résultats obtenus. Les flèches orientées vers le haut indiquent une augmentation de la variable. Les flèches orientées vers le bas indiquent une diminution de la variable. Le signe d'égalité (=) donnée (nombre variant de 0 à 1 , où 0 signifie l'égalité parfaite - tout le monde a le même revenu - et 1 signifie l'inégalité totale). Ensuite, le ratio $(10 \% / 10 \%)$ indique le ratio entre le total des revenus du $10 \%$ de la population la plus riche divisé par le total des revenus du $10 \%$ de la population la plus pauvre. Enfin, le ratio $(20 \% / 20 \%)$ indique le ratio entre le total des revenus du $20 \%$ de la population la plus riche divisé par le total des revenus du $20 \%$ de la population la plus pauvre. Par sa plus grande étendue, ce ratio est parfois plus réaliste.

Grâce à la spécification des indicateurs de commerce international, de développement, de pauvreté et d'inégalité, il a été possible de colliger les données ayant servi à l'analyse des relations entre les variables du modèle.

indique une stabilisation de la variable. Avant d'analyser la situation spécifique à chaque pays de l'échantillon, il est utile de signaler deux observations.

Tableau 2 : Résultats empiriques

\begin{tabular}{|c|c|c|c|c|c|c|c|c|c|c|}
\hline Pays & \multicolumn{2}{|c|}{ Commerce } & Dev. & \multicolumn{4}{|c|}{ Pauvreté. } & \multicolumn{3}{|c|}{ Inégalités } \\
\hline & 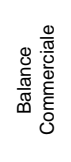 & 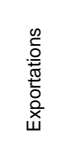 & 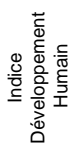 &  &  &  & 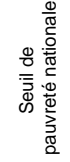 & 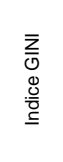 & 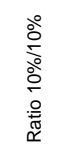 &  \\
\hline$\underline{\underline{a}}$ & & & & & $乃$ &  &  & 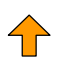 & & $E$ \\
\hline  & & & & & &  & 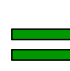 & 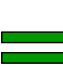 & 3 & \\
\hline 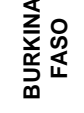 & &  & & & 凸 &  & $\bar{E}$ & & $\Omega$ & \\
\hline 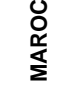 & & & & & & & $=$ & & & \\
\hline 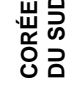 & & & & HPI-2 & 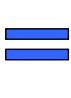 & $\bar{E}$ &  & & & \\
\hline
\end{tabular}


La première observation est que les statistiques disponibles pour les mesures d'inégalités sont rares et difficiles à trouver. Cela est probablement causé par l'énorme travail de récolte de données qui doit s'effectuer sur le terrain pour générer des statistiques fiables. Nous parlerons donc de ces statistiques avec précaution. La seconde observation est qu'une augmentation des exportations ne se traduit pas toujours par une augmentation de la balance commerciale puisque les importations rentrent en compte dans la détermination de la balance commerciale. Nous procédons ensuite à l'analyse des résultats pour chaque pays de l'échantillon.

\subsection{Le cas de l'Inde}

L'Inde est considéré comme un pays émergent très solide au niveau économique, mais très inégal au niveau de la répartition des richesses.

Graphique 1 : Évolution IDH



Graphique 3 : Évolution pauvreté

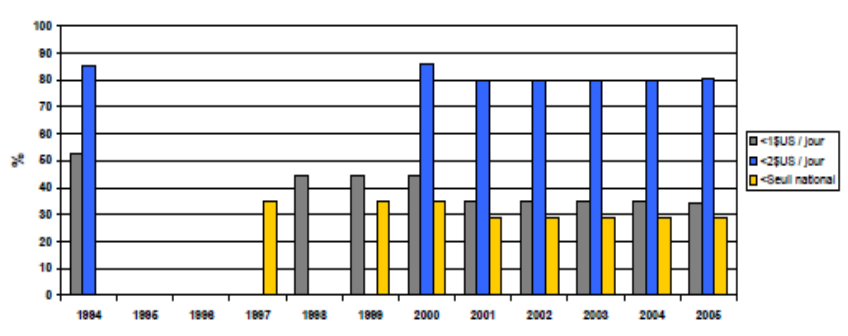

Dans le cas de l'Inde, les exportations et le développement ont augmenté. De plus, la pauvreté a diminué selon tous les indices existants. L'indice GINI indique cependant que les inégalités ont tout de même augmenté. En considérant que le développement a énormément augmenté et que la réduction de la pauvreté n'a pas été si intense, on peut probablement expliquer la répercussion sur l'indice GINI par un développement pas assez inclusif. Dans une optique de réduction de la pauvreté, la théorie semble être confirmée dans le cas de l'Inde, tel que présenté dans les graphiques 1 à 8 . C'est d'ailleurs pourquoi l'Inde est considéré comme un pays émergent très solide au niveau économique, mais très inégal au niveau de la répartition des richesses.

Graphique 2 : Évolution HPI-1

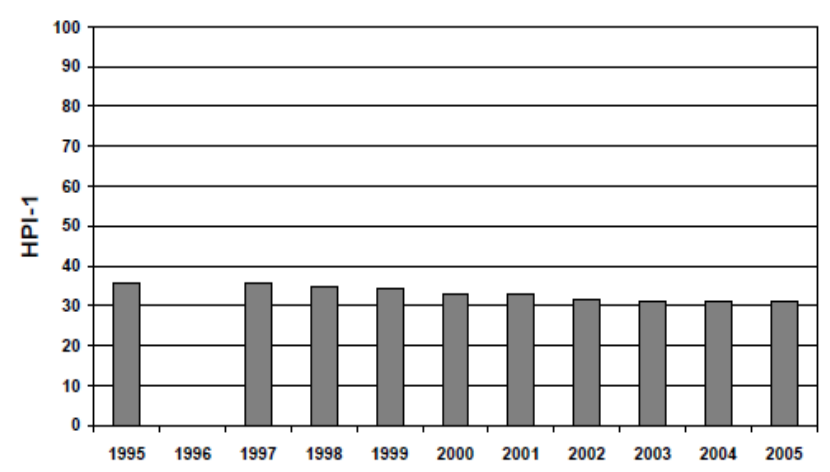

Graphique 4 : Évolution Indice de Gini

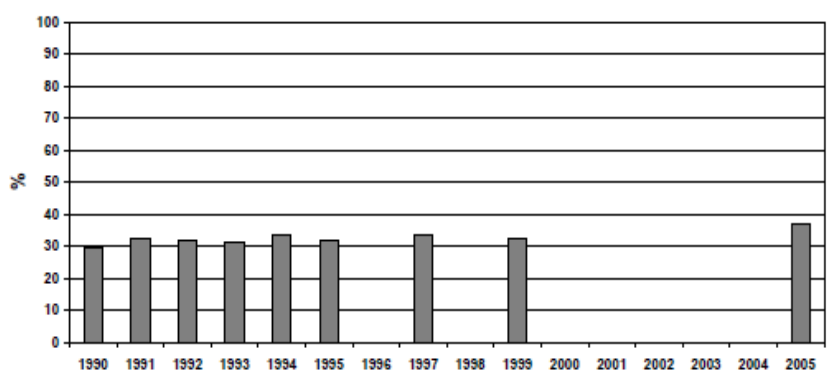


Graphique 5 : Évolution ratio 10\%/10\%



Graphique 7 : Évolution balance commerciale

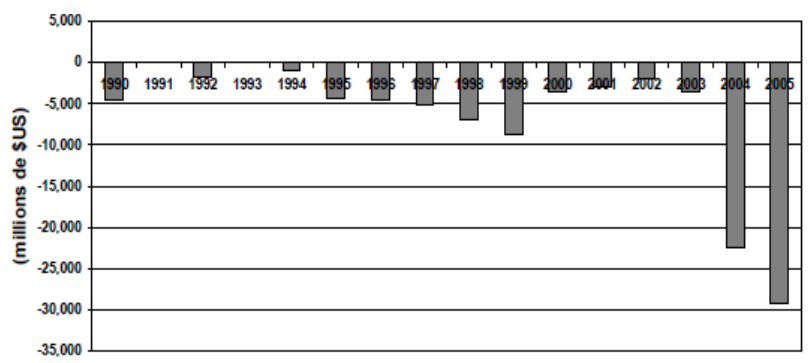

Graphique 6 : Évolution ratio $20 \% / 20 \%$



Graphique 8 : Évolution exportations

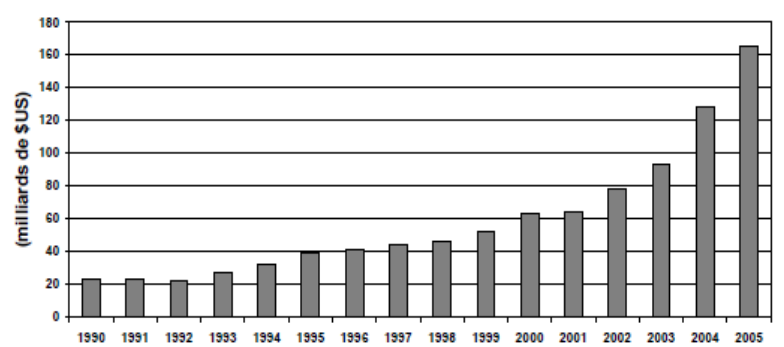

lation dans une pauvreté extrême. Grâce à ces données et en considérant le poids économique du Brésil, il convient de dire qu'il ne s'agit pas d'une pauvreté causée par la pauvreté généralisée qui sévit dans les Pays moins avancés, mais plutôt par des phénomènes sociaux ou une économie qui délaisse une portion très ciblée de sa population. Dans une optique de réduction de la pauvreté, le gouvernement brésilien aurait peut-être avantage à s'assurer que son développement inclut les plus pauvres. La théorie semble toutefois être confirmée (voir graphiques 9 à 16).

Graphique 10 : Évolution HPI-1

Graphique 11 : Évolution pauvreté

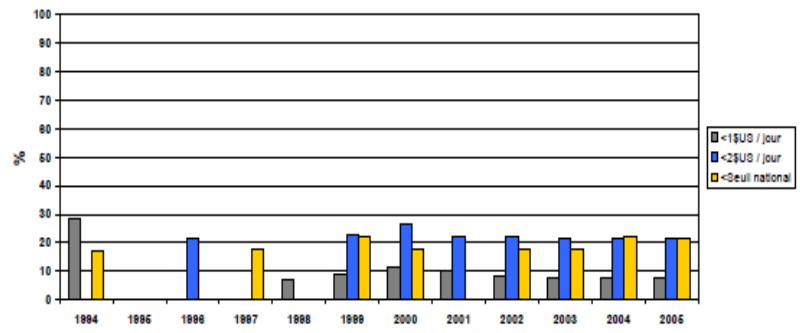

Graphique 13 : Évolution ratio 10\%/10\%



Graphique 15 : Évolution balance commerciale

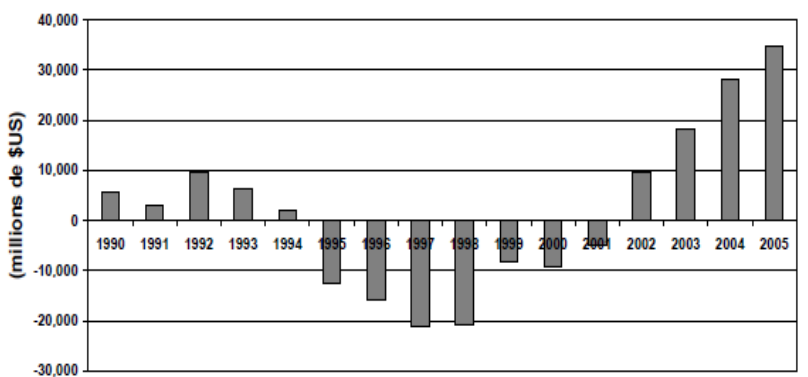

Graphique 12 : Évolution Indice de Gini



Graphique 14 : Évolution ratio 20\%/20\%



Graphique 16 : Évolution exportations - Brésil

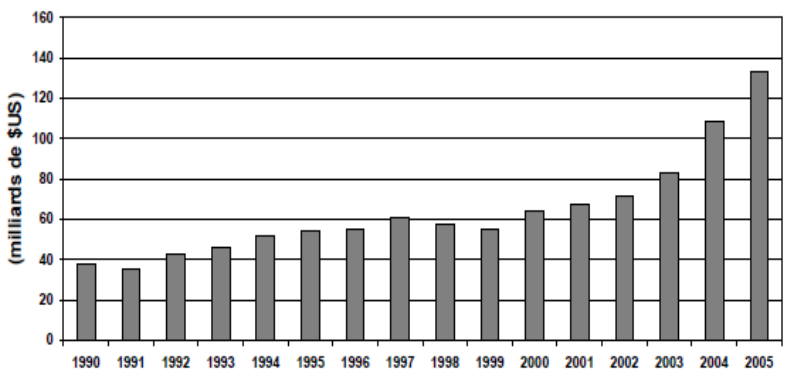

\subsection{Le cas du Burkina Faso}

\section{Au Burkina Faso, l'augmentation des exportations n'entraîne pas nécessairement un développement ayant un impact positif sur la pauvreté et les inégalités.}

Dans le cas du Burkina Faso, les exportations n'ont pas vraiment augmenté depuis 15 ans. Elles se situent toujours sous la barre de un milliard de dollars américains. L'indice de développement humain a augmenté, mais tous les indices de pauvreté sont restés stables ou ont diminué. Dans ce cas, les mesures d'inégalités ne sont pas très fiables. D'abord, l'augmentation de l'indice de développement humain, alors qu'il y a absence d'exportations pouvant générer une activité économique, peut s'expliquer par la réception annuelle et systématique de centaines de millions de dollars en aide internationale (voir graphiques 17 à 24). Ce pays attire très peu d'Indice de développement humain et figure au bas du palmarès mondial de l'investissement direct étranger. Des investissements dans les capacités de production et une ouverture accrue à l'exportation pourraient améliorer le sort du Burkina Faso. Par contre, d'importants questionnements demeurent. D'abord, en plus d'être frappé par la désertification au Nord, ce pays ne possède pas beaucoup de ressources de base comme l'eau potable. Ensuite, 
de grands efforts seraient à faire pour rectifier la mauvaise gouvernance et la corruption qui y sévit. Au Burkina Faso, l'augmentation des exportations n'entraîne pas nécessairement un développement ayant un impact positif sur la pauvreté et les inégalités.



Graphique 19 : Évolution pauvreté

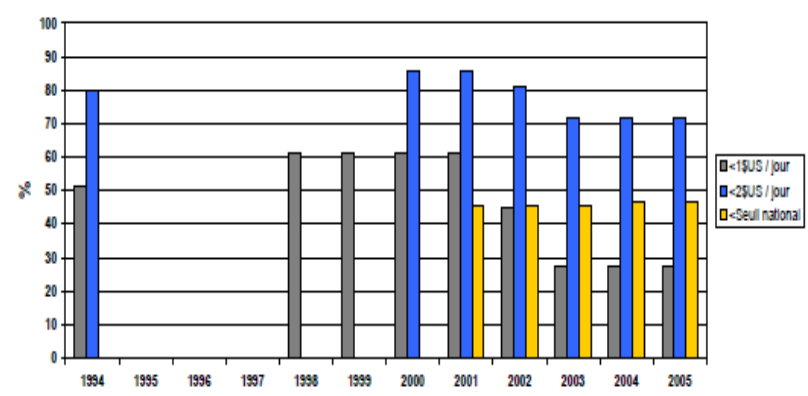

Graphique 21 : Évolution ratio 10\%/10\%

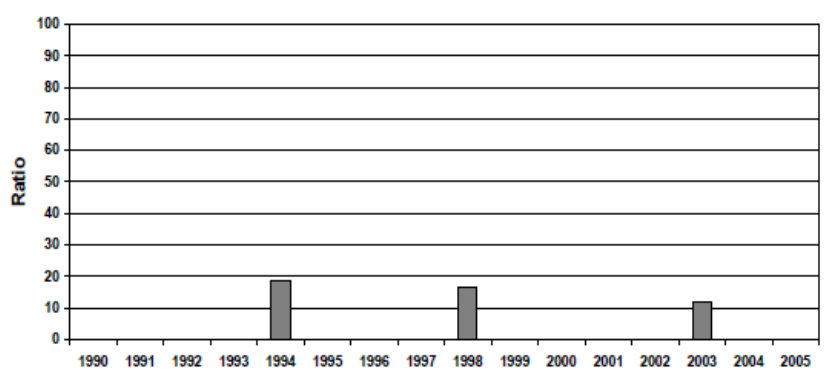

Graphique 23 : Évolution balance commerciale

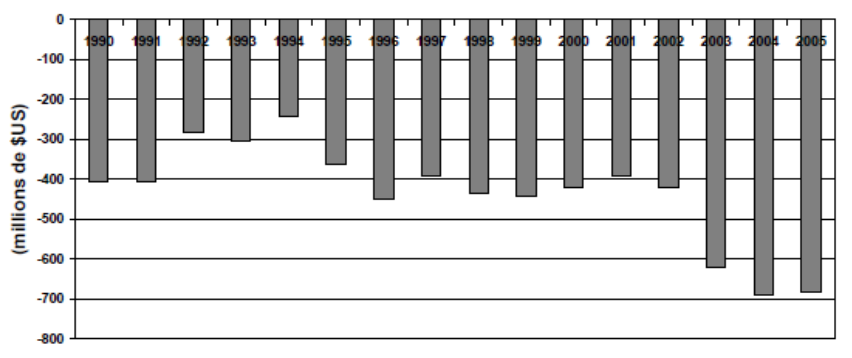

Des investissements dans les capacités de production et une ouverture accrue à l'exportation pourraient améliorer le sort du Burkina Faso.



Graphique 20 : Évolution Indice de Gini



$\begin{array}{llllllllllllllll}1990 & 1991 & 1992 & 1993 & 1994 & 1995 & 1996 & 1997 & 1998 & 1999 & 2000 & 2001 & 2002 & 2003 & 2004 & 2005\end{array}$

Graphique 22 : Évolution ratio $20 \% / 20 \%$

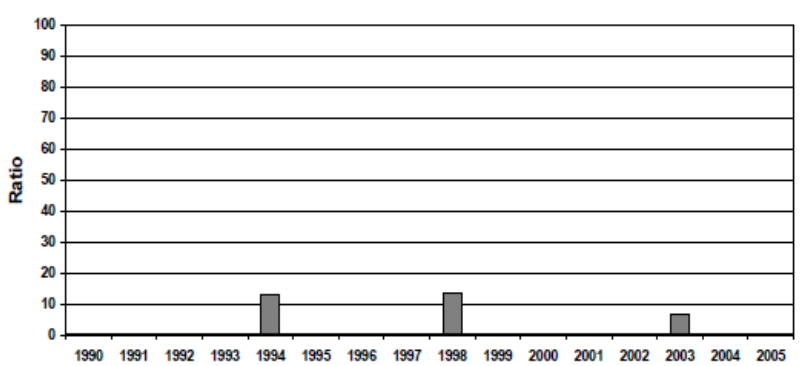

Graphique 24 : Évolution exportations






\subsection{Le cas du Maroc}

Dans le cas du Maroc, le commerce ainsi que l'indice du développement humain ont augmenté. Par contre, tous des indices de pauvreté sont restés stables à l'exception de l'indice HPI-1 qui a diminué. Les mesures d'inégalités pourraient aider à comprendre ce qui se passe, mais les données sont trop peu nombreuses et fiables. Une hausse du commerce international et du développement humain qui n'a pas d'impact sur les pauvres est considérée comme un développement non inclusif. Cette inclusion est une des caractéristiques nécessaires à la réduction de la pauvreté. On peut se douter

\section{Graphique 25 : Évolution IDH}

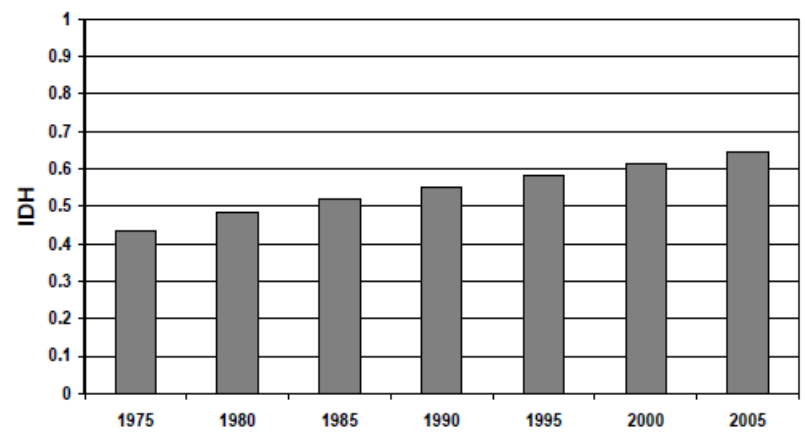

Graphique 27 : Évolution pauvreté

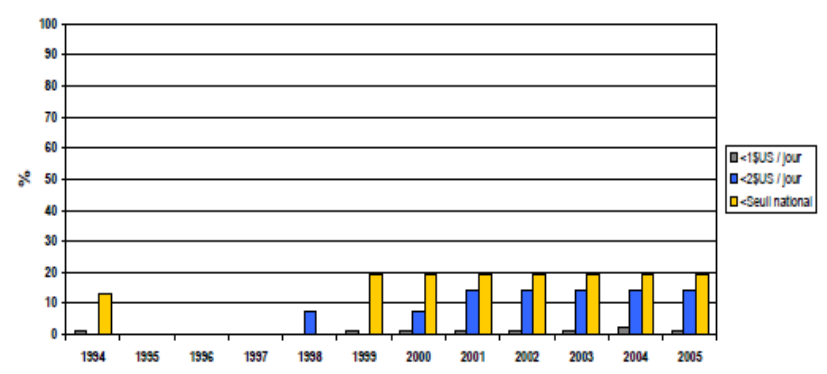

Graphique 29 : Évolution ratio 10\%/10\%

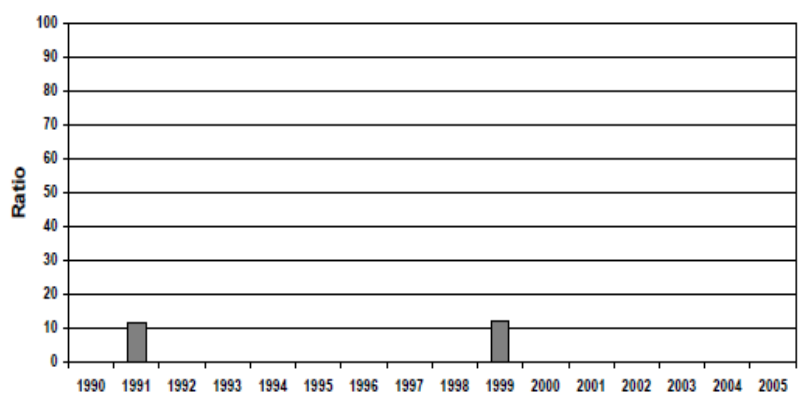

qu'en présence de données, les indices d'inégalités augmenteraient. Un exemple approprié est le fait que le tourisme dans les grandes villes $\mathrm{y}$ est en pleine expansion, alors que ce n'est pas le cas dans les campagnes. Une analyse poussée des mesures et des décisions gouvernementales serait nécessaire pour évaluer si les caractéristiques de croissance soutenue et inclusive y sont présentes. Dans le cas où elles sont absentes ou elles pourraient être améliorées, les conclusions de notre analyse supportent une fois de plus. Les hypothèses liées au modèle proposé dans le cadre de cette étude (voir graphiques 25 à 32).

Graphique 26 : Évolution HPI-1



Graphique 28 : Évolution Indice de Gini

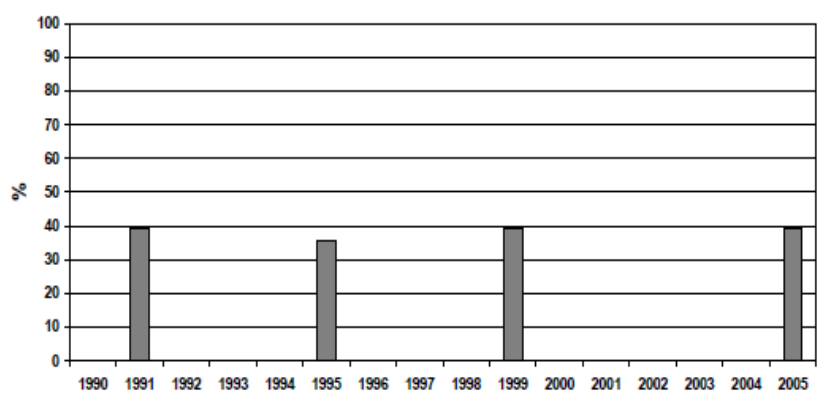

Graphique 30 : Évolution ratio $20 \% / 20 \%$




Graphique 31 : Évolution balance commerciale

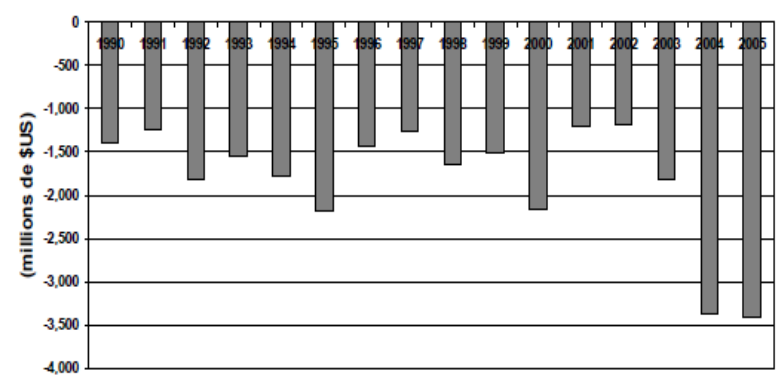

\subsection{Le cas de la Corée du Sud}

Le cas de la Corée du sud permet de conclure que le commerce entraine un développement qui a un impact positif sur la diminution de la pauvreté et les inégalités.

Dans le cas de la Corée du Sud, il y a une augmentation constante du commerce international depuis les années 1990. L'indice de développement humain apparaît corrélé au volume du commerce international. Maintenant, au niveau de nos indices de pauvreté, ils sont restés stables. Au début des années 1990, la Corée du Sud avait déjà quitté le seuil de pauvreté qui caractérisait bon nombre de pays de notre échantillon. En effet, ses exportations se chiffraient déjà à 75

\section{Graphique 33 : Évolution IDH}



Graphique 35 : Évolution Indice de Gini



Graphique 32 : Évolution exportations



milliards de dollars US. Or, comme nous l'avons expliqué, l'impact entre la croissance économique et la réduction de la pauvreté s'atténue jusqu'au point de s'annuler au fur et à mesure que la croissance économique augmente. Dans le cas de la Corée du Sud, les taux de pauvreté selon les deux seuils de l'ONU sont inférieurs à $2 \%$ depuis au moins 1998. Cette pauvreté résiduelle ne sera donc pas diminuée par une augmentation des exportations. Elle trouve ses causes ailleurs et pourra être ciblée grâce à des initiatives sociales nationales. En bref, le cas de la Corée du Sud permet de conclure que le commerce entraîne un développement qui a un impact positif sur la diminution de la pauvreté et les inégalités (voir graphiques 33 à 39).

\section{Graphique 34 : Évolution pauvreté -} Corée du Sud

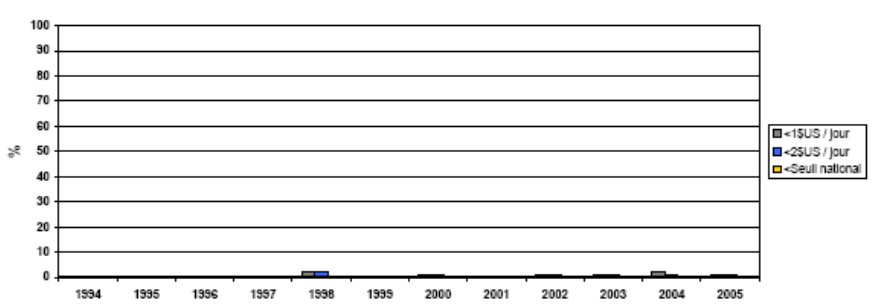

Graphique 36 : Évolution ratio 10\%/10\%

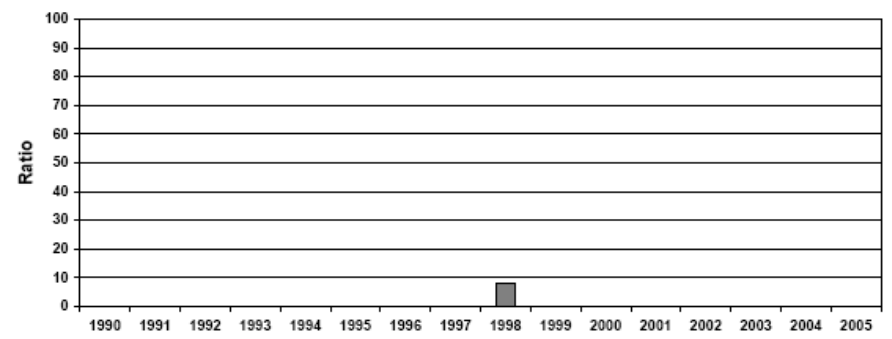


Graphique 37 : Évolution ratio 20\%/20\%



Graphique 38 : Évolution balance commerciale



Graphique 39 : Évolution exportations

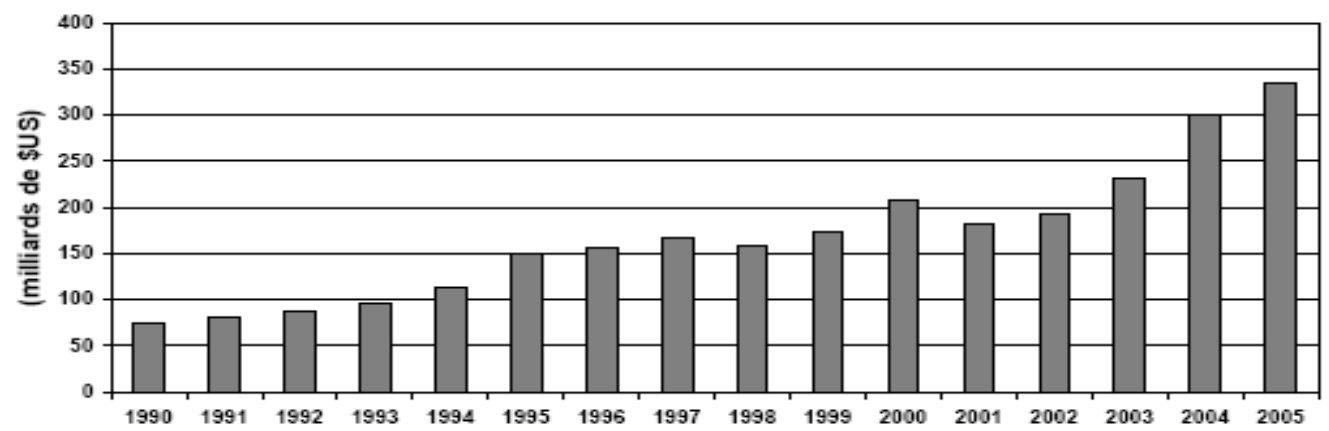

\section{CONCLUSION}

Parmi les cinq pays de notre échantillon, nous arrivons aux conclusions que pour trois pays faisant partie des nouveaux pays industrialisés (Inde, Brésil, Maroc) l'impact du commerce international sur la réduction de la pauvreté et des inégalités est important.

Le modèle d'analyse de l'impact du commerce international sur la réduction de la pauvreté et des inégalités proposé dans le cadre de cette étude s'avère plausible et réaliste. Parmi les cinq pays de notre échantillon, nous arrivons aux conclusions que pour trois pays faisant partie des nouveaux pays industrialisés (Inde, Brésil, Maroc) l'impact du commerce international sur la réduction de la pauvreté et des inégalités est important. Le cas de la Corée $\mathrm{du}$ Sud, un pays très avancé industriellement, confirme la théorie concernant la diminution de l'impact de la croissance économique sur la réduction de la pauvreté au fur et à mesure qu'elle augmente et supporte ainsi la nécessité de considérer séparément les pays développés des pays les moins avancés en matière de réduction de la pauvreté. Enfin, le cas du Burkina Faso, un pays en développement, démontre la nécessité de certaines conditions pour que le développement entraîne un impact positif sur la réduction de la pauvreté et des inégalités dans les pays les moins avancés.

L'augmentation du volume de commerce international peut donc avoir un impact sur la réduction de la pauvreté et des inégalités, mais cet impact n'est pas systématique. Il dépend des conditions de développement de base qui permettront d'avoir une croissance économique soutenue et inclusive. Il serait intéressant pour des recherches futures de tester l'hypothèse pour d'autres pays comme les pays membres de l'OPEP (Algérie, Arabie Saoudite, Angola, Nigeria, Venezuela, etc.) où les exportations de pétrole procurent une manne financière importante, mais qui ne permet pas de réduire considérablement la pauvreté et les inégalités.

L'augmentation du volume de commerce international peut donc avoir un impact sur la réduction de la pauvreté et des inégalités, mais cet impact n'est pas systématique. Il dépend des conditions de développement de base qui permettront d'avoir une croissance économique soutenue et inclusive. 


\section{BIBLIOGRAPHIE}

${ }^{1}$ Grusky D. B. et Kanbur R. (2006). «Poverty and Inequality », Stanford University Press

${ }^{2}$ Kohl, R. (2009). " Globalisation, Poverty and Inequality », OECD Publishing

${ }^{3}$ Winters, L. A. (2000). «Trade, Trade Policy and Poverty: What are the links? », London, Centre for Economic Policy Research.

${ }^{4}$ Conférence des Nations Unies sur le commerce et le développement (2004). «Rapport 2004 sur les pays les moins avancés: Commerce international et réduction de la pauvreté », Presse des Nations Unies. ${ }^{5}$ Sources des données : UNDP: Human Development Report, From 1995 to 2008; UNDP: HDR Stats (http://hdr.undp.org/en/statistics/); Perspectives http://perspective.usherbrooke.ca; World Bank, India Database; World Bank, World Development Indicators 2000; World Bank, World Development Indicators 2004; Socio-Economic Database for Latin America and the Caribbean, 2006; Deininger \& Squire, World Bank 2004; Szekely 2003; Szekely and Hilgert 2002; Ferreira and Litchfield 1996.

World Bank, Africa Department; World Bank Poverty Monitoring Database 2002; Cheong 2005; Lee 1991; Korea, NBS; http://www.atlas. iienetwork.org; http://www.wider.unu.edu

${ }^{6}$ Rapport sur le développement humain / Burkina Faso (2003), PNUD. 